\title{
The Role of Inflammation in the Pathogenesis of Colorectal Cancer
}

\author{
Beth B. McConnell, PhD and Vincent W. Yang, MD, PhD
}

\begin{abstract}
Chronic inflammatory disorders are often associated with an increased risk of developing cancer. A classic example of the connection between inflammation and cancer is the increased risk of colorectal cancer in patients with inflammatory bowel disease (IBD). In this review, we discuss aspects of IBD that promote colorectal cancer and highlight key molecular mediators that contribute to cancer risk. Additionally, we report on progress in identifying molecular targets that may prove efficacious in blocking the progression of IBD-related inflammation to cancer.
\end{abstract}

\section{Introduction}

Inflammatory bowel disease (IBD) affects approximately 1.4 million people in the United States. The most common forms of IBD are Crohn's disease (CD) and ulcerative colitis (UC). The primary differences between these diseases are the location and nature of the inflammatory response. CD can affect any part of the gastrointestinal tract and most commonly affects the terminal ileum, whereas UC is restricted to the colon and/or rectum. Furthermore, CD is characterized by granulomas and transmural inflammation, whereas UC-related inflammation is restricted to the mucosa and submucosa. Because of chronic damage to the colon and rectum in both types of IBD, these patients are at an increased risk of colorectal cancer (CRC). This risk is even more pronounced with longer duration of disease and greater extent and severity of colitis [1-3]. This review explores the relationship between inflammatory responses in IBD and the pathogenesis of CRC and discusses advances in understanding some of the key molecular culprits.

\section{Chronic Inflammation in IBD}

Chronic inflammatory responses that occur in IBD have been connected to predisposing genetic factors, which contribute to abnormal immune responses to luminal bacteria [4]. A number of genetic alterations associated with IBD have been identified in genes related to innate and adaptive immune responses. The first gene shown to be associated with CD was NOD2/ $C A R D 15$, which encodes a protein that acts as an intracellular pattern-recognition receptor for bacterial muramyl dipeptides [5,6]. Polymorphisms in two autophagy-related genes (ATG16L1 and IRGM) are also associated with CD and are important for degradation and antigen processing of intracellular pathogens [7,8]. Genes of the adaptive immune system that have been linked to CD and UC include components of the interleukin (IL)-23 signaling pathway, which affect T-cell differentiation. These are $I L 23 R$, which encodes a subunit of the receptor for IL-23R; IL12B, which encodes the p40 subunit of IL-12 and IL-23; and STAT3, which encodes signal transducer and activator of transcription $3[9,10]$. A working model for the pathogenesis of IBD is that genetic and environmental factors that predispose individuals to defects in sampling gut luminal antigens lead to activation of innate immune responses.

Corresponding author: Beth B. McConnell, PhD, Division of Digestive Diseases, Department of Medicine, Emory University School of Medicine, 201 Whitehead Research Building, 615 Michael Street, Atlanta, GA 30322, USA. E-mail: beth.mcconnell@emory.edu.

Disclosure: No potential conflicts of interest relevant to this article were reported. 
Exacerbated responses to antigens activate dendritic cells, which then mediate differentiation of naive $\mathrm{T}$ cells into effector cells [11]. In $\mathrm{CD}$, aberrant immune responses cause a bias toward the production of cytokines associated with a Thelper cell $1\left(\mathrm{TH}_{1}\right)$ and $\mathrm{TH}_{17}$ response. In contrast, UC disease is primarily associated with a $\mathrm{TH}_{2}$ and natural killer T-cell response [11]. Thus, the chronic activation of these key immune cells and their secreted products contribute to a tissue environment that can culminate in cancer.

\section{Key Players in Inflammation-Associated CRC}

In recent years, several molecules have emerged as critical players in contributing to inflammation-associated CRC. These culprits have been the focus of intense research to understand the molecular basis of their association with disease and to explore therapeutic possibilities. In this article, we highlight some of these molecules, namely cyclooxygenase- 2 (COX-2), nuclear factor- $\kappa \mathrm{B}$ (NF- $\mathrm{\kappa B})$, tumor necrosis factor- $\alpha$ (TNF- $\alpha$ ), and toll-like receptors (TLRs), describing their connection to inflammation and cancer and discussing recent advances in elucidating their contribution to disease.

\section{COX-2}

COX-2 is an intermediate response gene that encodes a cytoplasmic protein, which catalyzes the synthesis of prostaglandins from arachidonic acid. COX-2 expression is activated by numerous proinflammatory cytokines, including IL- $1 \alpha$, IL- $1 \beta$, and TNF- $\alpha$, and it is increased at sites of inflammation [12]. In relation to human cancer, COX-2 is overexpressed in approximately $80 \%$ of CRCs and $40 \%$ of colorectal adenomas relative to normal mucosa [13]. By immunohistochemistry, COX-2 is found in the cytoplasm of neoplastic colonic epithelial cells and to a lesser extent in stromal cells, whereas normal epithelium is negative for COX-2 [14]. Activation of COX-2 expression in neoplastic cells is also seen in genetic mouse models of intestinal cancer involving mutations in the tumor suppressor adenomatous polyposis coli $(A p c)$ and in rodent models of colon cancer using a combination treatment of azoxymethane (AOM) and dextran sodium sulfate (DSS) to induce inflammation-associated tumors [14].

The contribution of COX-2 to the development of CRC is further supported by the fact that coxibs - a class of nonsteroidal anti-inflammatory drugs that specifically target COX-2 - are highly effective in reducing colon cancer in rodent models and humans $[15,16]$. COX-2 is thought to contribute to tumor development by modulating apoptosis, angiogenesis, and tumor invasiveness. Effects on apoptosis were shown in rat intestinal epithelial cells with ectopic expression of COX-2 [17]. These cells had increased adherence to the extracellular matrix, resistance to apoptosis-inducing agents, and upregulation of the antiapoptotic protein B-cell lymphoma-2 (Bcl-2). Similar results were seen with overexpression of COX-2 in human colon cancer cells in which COX-2 provided resistance to apoptosis following treatment with antineoplastic drugs [18]. In affecting the progression of cancer, COX-2 has been shown to increase invasiveness of colon cancer cells by way of activation of metalloproteinase- 2 (MMP-2) [19]. Furthermore, suppression of COX-2 in human prostate tumor cells by a selective COX-2 inhibitor resulted in reduced levels of MMP-2 and MMP-9 [20]. In relation to angiogenesis, expression of COX-2 in Caco-2 and HCA-7 colon cancer cells correlated with high levels of vascular endothelial growth factor, basic fibroblast growth factor, and endothelin-1, which stimulate endothelial migration and endothelial tube formation [21].

Given the extensive role of COX-2 in inflammation and various aspects of carcinogenesis, COX-2 is an attractive target for treatment of inflammatory disorders and cancer. A search for COX-2-specific inhibitors resulted in promising candidates, such as valdecoxib, celecoxib, and rofecoxib. Indeed, clinical trials with specific COX-2 inhibitors showed great promise for 
treatment of patients with prior adenomas. However, these trials were discontinued because of increased risk of thrombotic events $[22 \bullet \bullet, 23 \bullet \bullet]$.

\section{NF-KB}

NF- $\mathrm{\kappa B}$ is a key regulator of innate immunity and inflammation, and its constitutive activation is associated with various types of cancer, including acute lymphoblastoid leukemia, Hodgkin's lymphoma, and breast, ovarian, prostate, and colon cancer [24]. NF- $\mathrm{BB}$ is a dimeric transcription factor that is composed of members of the Rel transcription factor family, and its activity is directed toward a wide array of genes that promote proliferation and survival [25]. In tumor, epithelial, and immune cells, NF- $\mathrm{kB}$ activates the expression of genes encoding inflammatory cytokines, adhesion molecules, enzymes in the prostaglandin synthesis pathway (including COX-2), inducible nitric oxide synthase, and angiogenic factors [26••]. In addition, $\mathrm{NF}-\kappa \mathrm{B}$ induces expression of a number of antiapoptotic genes, including Bcl-2 family members, which can provide a survival advantage to precancerous or tumor cells.

In the resting nonstimulated state, $\mathrm{NF}-\mathrm{\kappa B}$ dimers are cytoplasmic and inactive. Activation of NF- $\kappa B$ occurs downstream from signaling pathways mediated through TLRs and other microorganism-sensing molecules or by the inflammatory cytokines TNF- $\alpha$ and IL-1 $\beta$ [26••]. The canonical regulation of NF- $\mathrm{KB}$ involves sequestration of NF- $\mathrm{KB}$ in the cytoplasm by the

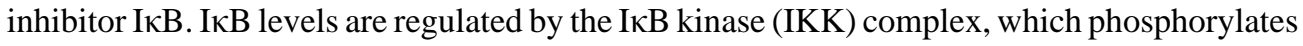
$\mathrm{I} \kappa \mathrm{B}$, targeting it for ubiquitin-dependent degradation [27]. Upon the degradation of I $\mathrm{KB}, \mathrm{NF}-$ $\kappa \mathrm{B}$ is released and translocates to the nucleus, where it becomes transcriptionally active. Targets of NF- $\mathrm{KB}$ can be separated into four functional categories: antiapoptotic genes, inflammatory and immunoregulatory genes, genes that promote cell cycle progression, and genes that encode negative regulators of NF- $\mathrm{KB}$. Many of these targets have been associated with cancer initiation and progression [27].

A seminal article presented by Greten et al. [28] in which NF- $\mathrm{KB}$ activity was disrupted in a mouse model of colitis-associated cancer contributes to our understanding of the role of NF$\kappa \mathrm{B}$ in inflammation-associated carcinogenesis. In this article, the authors developed mice with deletions of IKK $\beta$, a component of the IKK complex, in intestinal epithelial cells or myeloid cells, resulting in functional inactivation of NF- $\mathrm{KB}$ in the two cell types. The authors then tested the effects of inactivation of NF- $\mathrm{kB}$ in the two cell types by treating the mice with a combination of AOM and DSS to induce inflammation-associated tumors. When NF- $\mathrm{KB}$ activity was disrupted in colonic epithelial cells, there was a dramatic reduction in tumor number in these mice. In examining the mechanism, there was enhanced epithelial cell apoptosis during early tumor development without any reduction in inflammation. Thus, the authors concluded that activation of NF- $\mathrm{KB}$ in epithelial cells contributes to tumor initiation and promotion primarily by suppressing apoptosis; when that suppression is removed, precancerous cells are eliminated through cell death mechanisms. In mice with IKK $\beta$ deleted in the myeloid cells, there was reduced expression of many genes involved in inflammatory responses, including IL- $1 \beta$, IL-6, macrophage inflammatory protein 2 (MIP-2), TNF- $\alpha, \mathrm{COX}-2$, and intercellular adhesion molecule (ICAM) [28]. These mice exhibited a significant reduction in tumor size and some reduction in tumor number. From these results, the authors suggest that myeloid cells contribute to tumor development through production of paracrine signaling molecules that promote tumor growth.

In addition to promoting proliferation and providing protection from apoptosis, NF- $\mathrm{kB}$ has been shown in a number of studies to enhance invasion of tumor cells through regulating genes that promote metastasis and angiogenesis [25]. Evidence for NF- $\mathrm{KB}$ signaling affecting metastatic potential is found in an article by Luo et al. [29], who used a prostate cancer model to show that inhibition of IKK $\beta$ activation slowed cancer growth and inhibited metastatogenesis. Activation of IKK $\beta$ inhibited expression of the metastasis suppressor gene 
Maspin, thus promoting the metastatic phenotype. NF- $\mathrm{kB}$ has also been shown to induce vascular endothelial growth factor and COX-2, which promote angiogenesis [27].

Given the role of constitutive NF- $\mathrm{kB}$ activity in promoting tumor initiation and progression, the question arises whether NF- $\mathrm{kB}$ can be targeted for anti-inflammatory and/or cancer prevention therapies. In contrast to its pathogenic roles, NF- $\mathrm{KB}$ is clearly important for promoting innate immune responses to infection, and its absence may result in severe immunodeficiency. Thus, prolonged or significant inhibition of NF- $\mathrm{KB}$ may not be a practical

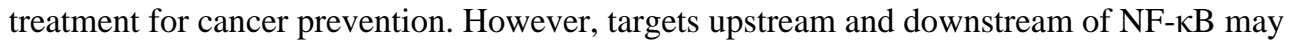
provide potential therapies.

\section{TNF- $\alpha$}

TNF- $\alpha$ is a member of the TNF superfamily of cytokines and is a key molecule regulating inflammation and host defense. Activation of TNF receptors (TNFRs) leads to the recruitment of intracellular adaptor proteins, which in turn activate several distinct signal transduction pathways [30]. Binding of TNF- $\alpha$ to its receptors has two primary outcomes-activation of inflammatory/cell survival pathways through NF- $\mathrm{\kappa B}$ or activation of apoptotic pathways through caspase 8 .

TNF- $\alpha$ has been shown to have conflicting roles in cancer, serving as a tumor-promoting or tumor-destructive factor [31]. The latter role is observed as hemorrhagic tumor necrosis, which occurs through selective destruction of tumor blood vessels and activation of T cells that attack and eliminate tumor cells. However, this antitumor activity requires high levels of TNF- $\alpha$, which can be extremely toxic. At low doses, chronic production of TNF- $\alpha$ is a feature of many tumor types and can promote all stages of cancer development, from growth to invasion and metastasis. Several clinical studies provide evidence for the contribution of TNF- $\alpha$ to the development of CRCs and other human cancers. For example, TNF- $\alpha$ expression is found in the tumor microenvironment of many tumor types, including breast, ovarian, colorectal, prostate, melanoma, lymphomas, and leukemia [32]. In addition, TNF- $\alpha$ mRNA and protein levels are increased to abnormal levels in preneoplastic Helicobacter pylori-positive gastric lesions and inflamed colonic mucosa [33].

Popivanova et al. [34••] recently explored the contribution of TNF- $\alpha$ signaling to the development of CRC using a genetic mouse model deficient in type 1 TNFR-p55. The authors found that abrogation of TNF- $\alpha$ signaling in mice significantly reduced colitis following AOM and DSS treatment, with decreased mucosal damage, inflammatory cell infiltrates, and cytokine expression in the mucosa. These effects corresponded with markedly reduced tumor formation. The contribution of TNF- $\alpha$ to inflammation-associated cancer was further tested by administering etanercept, a specific antagonist of TNF- $\alpha$, to wild-type mice following treatment with AOM and DSS. This treatment also suppressed neutrophil and macrophage infiltration into the mucosa and reduced the number and size of tumors. Etanercept is approved for treatment of rheumatoid arthritis but lacks the efficacy of anti-TNF- $\alpha$ monoclonal antibodies, such as infliximab, in treating IBD. However, given the results of this study, it is highly possible that monoclonal antibodies directed against TNF- $\alpha$ that are being used for clinical treatment of IBD will have a similar ability to inhibit tumor formation in these patients [35].

In addition to TNF- $\alpha$, a number of other inflammatory cytokines and adhesion molecules are being targeted as potential therapies for IBD [36•], and these may also be efficacious for the prevention and treatment of CRC. Therapies in clinical trials include antibodies against the p40 subunit of IL-12/IL-23 (ABT-874), the IL-6 receptor (MRA), interferon- $\gamma$ (fontolizumab), and the IL-2 receptor (CD25). In addition, adhesion molecules that promote trafficking of leukocytes into the inflamed gut wall, such as $\alpha_{4}$-integrins, vascular cellular adhesion molecule 
1 (VCAM-1), and ICAM-1 are being targeted with antibodies and oligodeoxynucleotides. The challenge of these strategies is to identify therapies that can reduce aberrant inflammatory responses while retaining proper defenses against infection and functional tumor surveillance mechanisms.

\section{TLRs}

TLRs play a major role in sensing gut microbiota by the intestinal mucosa, and activation of these receptors is required to maintain intestinal homeostasis. This homeostasis is often disturbed in IBD, in which abnormal immune responses to luminal bacteria play a key role in disease pathogenesis. Several polymorphisms in genes encoding innate immune receptors have now been associated with IBD through linkage analysis and population-based association studies. As mentioned previously, the first susceptibility gene associated with $\mathrm{CD}$, NOD2/ CARD15, encodes a protein that acts as an intracellular pattern-recognition receptor for the bacterial component muramyl dipeptide [5]. In addition, polymorphisms in TLR4, a receptor required for innate immunity to gram-negative bacteria, have been associated with UC and CD [37•]. TLR4 is upregulated in intestinal epithelial cells in active CD and UC [38], and TLR4 signaling has been shown to induce production of inflammatory molecules, such as COX-2, prostaglandin $\mathrm{E}_{2}$, and reactive oxygen species [37•].

In addition to the involvement of TLRs in IBD, evidence is mounting to support a role for TLRs in carcinogenesis. Recently, it was shown that a deficiency in the TLR adaptor, MyD88, significantly reduced tumor number and size in the $A p c^{\mathrm{min} /+}$ mouse model of intestinal tumorigenesis [39]. Furthermore, the absence of bacteria in several mouse models of inflammation-associated cancer has resulted in abrogation of dysplasia or cancer [37•]. Thus, the presence and recognition of bacteria in the gut appear necessary for inflammationassociated carcinogenesis. Examples of genetic alterations of TLRs in cancer include polymorphisms in TLR4 and TLR10 that have been associated with prostate cancer, and $T L R 4$ polymorphisms that have been identified in gastric cancer [40]. In specifically examining the contribution of TLR4 to carcinogenesis, mice inoculated with colon cancer cells silenced for expression of TLR4 showed increased survival and a significant reduction in tumor size compared with mice injected with cells expressing control short interfering RNA (siRNA) [41]. Furthermore, using the AOM-DSS mouse model of CRC, it was shown that TLR4deficient mice exhibited significantly reduced tumor number and size compared with wildtype controls [42].

An important end point of TLR signaling is NF- $\mathrm{KB}$ activation, and aberrant TLR signaling may contribute to the tumor-promoting activity of NF- $\mathrm{kB}$. Thus, specific TLR pathways may provide new targets for therapies to interrupt oncogenic pathways associated with IBD.

\section{Conclusions}

The connection between inflammation and cancer is becoming generally accepted, and the transcription factor NF- $\mathrm{KB}$ has emerged as a key mediator of this link. Given its comprehensive role in promoting inflammation-associated cancer, the NF- $\kappa B$ pathway provides a promising target for cancer therapy. Supporting this idea, numerous anti-inflammatory and anticancer drugs, including nonsteroidal anti-inflammatory drugs, glucocorticoids, and anti-inflammatory cytokines demonstrate the ability to block NF- $\kappa B$ activation [24]. NF- $\kappa B$ induces the expression of powerful proinflammatory mediators, such as COX-2 and TNF- $\alpha$; these, along with other transcription targets, provide numerous possibilities for treatment for IBD and colitis-associated cancer. With continued progress in understanding the contribution of various cytokines to the pathogenesis of inflammation-associated cancer, the use of cytokine antagonists as anti-inflammatory treatment is a growing area. Signaling molecules upstream 
of NF- $\kappa \mathrm{B}$ may likewise provide attractive targets for therapies, such as molecules involved in recognition of gut microbiota for inflammation-associated CRC.

In examining the functional attributes of the molecules discussed in this review, what becomes evident is their interconnectedness (Fig. 1). From signaling molecules to transcription factors to downstream targets, the complexity of inflammatory pathways and mechanisms only increases as we continue to make discoveries. Like pulling a thread and unraveling the fabric, targeted therapies can have unexpected outcomes and side effects. However, given the success of some of these therapies, such as anti-TNF- $\alpha$ treatment for chronic inflammatory diseases, pulling the correct thread can prove very effective.

\section{References and Recommended Reading}

Papers of particular interest, published recently, have been highlighted as:

- Of importance

•• Of major importance

1. Gupta RB, Harpaz N, Itzkowitz S, et al. Histologic inflammation is a risk factor for progression to colorectal neoplasia in ulcerative colitis: a cohort study. Gastroenterology 2007;133:1099-1105. [PubMed: 17919486]

2. Itzkowitz SH, Harpaz N. Diagnosis and management of dysplasia in patients with inflammatory bowel diseases. Gastroenterology 2004;126:1634-1648. [PubMed: 15168373]

3. Rutter M, Saunders B, Wilkinson K, et al. Severity of inflammation is a risk factor for colorectal neoplasia in ulcerative colitis. Gastroenterology 2004;126:451-459. [PubMed: 14762782]

4. Sands BE. Inflammatory bowel disease: past, present, and future. J Gastroenterol 2007;42:16-25. [PubMed: 17322989]

5. Hugot JP, Chamaillard M, Zouali H, et al. Association of NOD2 leucine-rich repeat variants with susceptibility to Crohn's disease. Nature 2001;411:599-603. [PubMed: 11385576]

6. Ogura Y, Bonen DK, Inohara N, et al. A frameshift mutation in NOD2 associated with susceptibility to Crohn's disease. Nature 2001;411:603-606. [PubMed: 11385577]

7. Hampe J, Franke A, Rosenstiel P, et al. A genome-wide association scan of nonsynonymous SNPs identifies a susceptibility variant for Crohn disease in ATG16L1. Nat Genet 2007;39:207-211. [PubMed: 17200669]

8. Parkes M, Barrett JC, Prescott NJ, et al. Sequence variants in the autophagy gene IRGM and multiple other replicating loci contribute to Crohn's disease susceptibility. Nat Genet 2007;39:830-832. [PubMed: 17554261]

9. Fisher SA, Tremelling M, Anderson CA, et al. Genetic determinants of ulcerative colitis include the ECM1 locus and five loci implicated in Crohn's disease. Nat Genet 2008;40:710-712. [PubMed: 18438406]

10. Franke A, Balschun T, Karlsen TH, et al. Sequence variants in IL10, ARPC2 and multiple other loci contribute to ulcerative colitis susceptibility. Nat Genet 2008;40:1319-1323. [PubMed: 18836448]

11. Shih DQ, Targan SR. Immunopathogenesis of inflammatory bowel disease. World J Gastroenterol 2008;14:390-400. [PubMed: 18200661]

12. Herschman HR, Xie W, Reddy S. Inflammation, reproduction, cancer and all that.... The regulation and role of the inducible prostaglandin synthase. Bioessays 1995;17:1031-1037. [PubMed: 8634064]

13. Eberhart CE, Coffey RJ, Radhika A, et al. Up-regulation of cyclooxygenase 2 gene expression in human colorectal adenomas and adenocarcinomas. Gastroenterology 1994;107:1183-1188. [PubMed: 7926468]

14. Sinicrope FA, Gill S. Role of cyclooxygenase-2 in colorectal cancer. Cancer Metastasis Rev 2004;23:63-75. [PubMed: 15000150] 
15. Jacoby RF, Seibert K, Cole CE, et al. The cyclooxygenase-2 inhibitor celecoxib is a potent preventive and therapeutic agent in the min mouse model of adenomatous polyposis. Cancer Res 2000;60:50405044. [PubMed: 11016626]

16. Oshima M, Murai N, Kargman S, et al. Chemoprevention of intestinal polyposis in the Apcdelta716 mouse by rofecoxib, a specific cyclooxygenase-2 inhibitor. Cancer Res 2001;61:1733-1740.

[PubMed: 11245490]

17. Tsujii M, DuBois RN. Alterations in cellular adhesion and apoptosis in epithelial cells overexpressing prostaglandin endoperoxide synthase 2. Cell 1995;83:493-501. [PubMed: 8521479]

18. Sun Y, Tang XM, Half E, et al. Cyclooxygenase-2 overex-pression reduces apoptotic susceptibility by inhibiting the cytochrome c-dependent apoptotic pathway in human colon cancer cells. Cancer Res 2002;62:6323-6328. [PubMed: 12414664]

19. Li G, Yang T, Yan J. Cyclooxygenase-2 increased the angiogenic and metastatic potential of tumor cells. Biochem Biophys Res Commun 2002;299:886-890. [PubMed: 12470662]

20. Attiga FA, Fernandez PM, Weeraratna AT, et al. Inhibitors of prostaglandin synthesis inhibit human prostate tumor cell invasiveness and reduce the release of matrix metalloproteinases. Cancer Res 2000;60:4629-4637. [PubMed: 10969817]

21. Tsujii M, Kawano S, Tsuji S, et al. Cyclooxygenase regulates angiogenesis induced by colon cancer cells. Cell 1998;93:705-716. [PubMed: 9630216]Cell 1998;94:271.Published erratum appears in

$22 \bullet$. Arber N, Kuwada S, Leshno M, et al. Sporadic adenomatous polyp regression with exisulind is effective but toxic: a randomised, double blind, placebo controlled, dose-response study. Gut 2006;55:367-373. [PubMed: 16150858]This article reports on the cardiac complications associated with this COX-2-specific inhibitor.

23••. Bertagnolli MM, Eagle CJ, Zauber AG, et al. Celecoxib for the prevention of sporadic colorectal adenomas. N Engl J Med 2006;355:873-884. [PubMed: 16943400]This report highlights the toxicity involved with COX-2 inhibitors.

24. Li Q, Withoff S, Verma IM. Inflammation-associated cancer: NF-kappaB is the lynchpin. Trends Immunol 2005;26:318-325. [PubMed: 15922948]

25. Naugler WE, Karin M. NF-kappaB and cancer-identifying targets and mechanisms. Curr Opin Genet Dev 2008;18:19-26. [PubMed: 18440219]

26••. Mantovani A, Allavena P, Sica A, Balkwill F. Cancer-related inflammation. Nature 2008;454:436444. [PubMed: 18650914]This is a thorough and insightful review that presents the role of inflammation in the malignant progression of epithelial cancers. It also addresses unanswered questions regarding cancer-related inflammation.

27. Greten FR, Karin M. The IKK/NF-kappaB activation pathway-a target for prevention and treatment of cancer. Cancer Lett 2004;206:193-199. [PubMed: 15013524]

28. Greten FR, Eckmann L, Greten TF, et al. IKKbeta links inflammation and tumorigenesis in a mouse model of colitis-associated cancer. Cell 2004;118:285-296. [PubMed: 15294155]

29. Luo JL, Tan W, Ricono JM, et al. Nuclear cytokine-activated IKKalpha controls prostate cancer metastasis by repressing Maspin. Nature 2007;446:690-694. [PubMed: 17377533]

30. Balkwill F. TNF-alpha in promotion and progression of cancer. Cancer Metastasis Rev 2006;25:409416. [PubMed: 16951987]

31. Szlosarek P, Charles KA, Balkwill FR. Tumour necrosis factor-alpha as a tumour promoter. Eur J Cancer 2006;42:745-750. [PubMed: 16517151]

32. Szlosarek PW, Balkwill FR. Tumour necrosis factor alpha: a potential target for the therapy of solid tumours. Lancet Oncol 2003;4:565-573. [PubMed: 12965278]

33. Noguchi M, Hiwatashi N, Liu Z, Toyota T. Secretion imbalance between tumour necrosis factor and its inhibitor in inflammatory bowel disease. Gut 1998;43:203-209. [PubMed: 10189845]

34••. Popivanova BK, Kitamura K, Wu Y, et al. Blocking TNF-alpha in mice reduces colorectal carcinogenesis associated with chronic colitis. J Clin Invest 2008;118:560-570. [PubMed: 18219394 This article highlights the promise of anti-TNF- $\alpha$ treatments to reduce colorectal carcinogenesis.

35. Burstein E, Fearon ER. Colitis and cancer: a tale of inflammatory cells and their cytokines. J Clin Invest 2008;118:464-467. [PubMed: 18219390] 
36• Nakamura K, Honda K, Mizutani T, et al. Novel strategies for the treatment of inflammatory bowel disease: selective inhibition of cytokines and adhesion molecules. World J Gastroenterol 2006;12:4628-4635. [PubMed: 16937430]This article provides an overview of clinical trials underway that utilize therapies targeting cytokines and adhesion molecules and describe strategies and outcomes.

37•. Fukata M, Abreu MT. Role of Toll-like receptors in gastrointestinal malignancies. Oncogene 2008;27:234-243. [PubMed: 18176605]This review brings to light growing evidence for a connection between abnormal activation of TLRs and gastrointestinal cancer.

38. Cario E, Podolsky DK. Differential alteration in intestinal epithelial cell expression of toll-like receptor 3 (TLR3) and TLR4 in inflammatory bowel disease. Infect Immun 2000;68:7010-7017. [PubMed: 11083826]

39. Rakoff-Nahoum S, Medzhitov R. Regulation of spontaneous intestinal tumorigenesis through the adaptor protein MyD88. Science 2007;317:124-127. [PubMed: 17615359]

40. El-Omar EM, Ng MT, Hold GL. Polymorphisms in tollike receptor genes and risk of cancer. Oncogene 2008;27:244-252. [PubMed: 18176606]

41. Huang B, Zhao J, Li H, et al. Toll-like receptors on tumor cells facilitate evasion of immune surveillance. Cancer Res 2005;65:5009-5014. [PubMed: 15958541]Cancer Res 2005;65:9108.Published erratum appears in

42. Fukata M, Chen A, Vamadevan AS, et al. Toll-like receptor-4 promotes the development of colitisassociated colorectal tumors. Gastroenterology 2007;133:1869-1881. [PubMed: 18054559] 


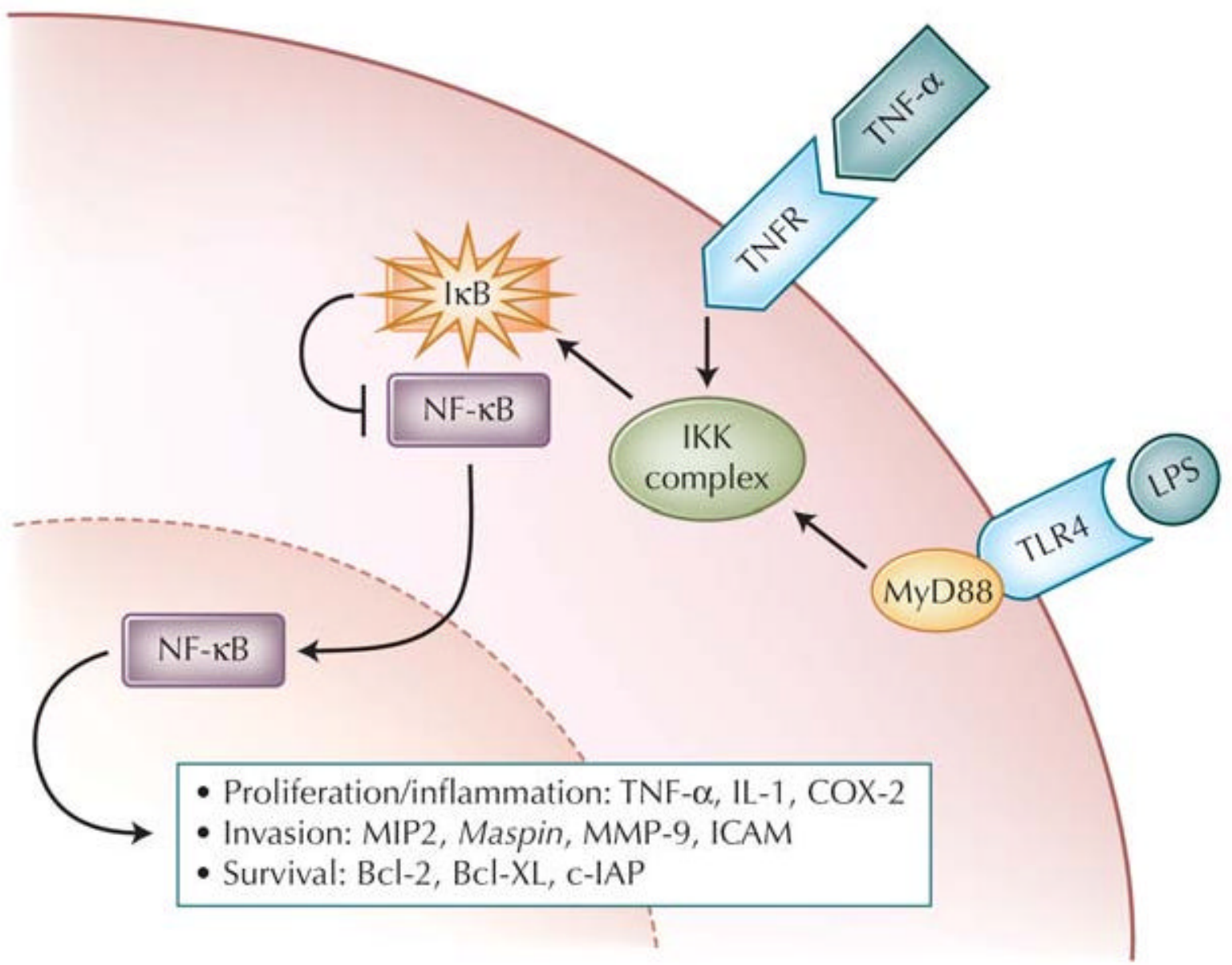

Figure 1.

Key mediators of inflammation-related colorectal cancer. Tumor necrosis factor- $\alpha$ (TNF- $\alpha)$ and bacterial components for toll-like receptors (TLRs) activate nuclear factor- $\kappa \mathrm{B}(\mathrm{NF}-\kappa \mathrm{B})$ through the I $\kappa \mathrm{B}$ kinase (IKK) complex. NF- $\kappa \mathrm{B}$ in turn induces transcription of genes involved in several aspects of tumorigenesis. Molecular targets, such as TNF- $\alpha$ and cyclooxygenase- 2 (COX-2), support and enhance inflammatory responses. Chronic activation of these pathways in the gut provides an environment that promotes the establishment of colorectal cancer. Bcl-2 -B-cell lymphoma-2; Bcl-XL_-basal cell lymphoma-extra large; c-IAP_cellular inhibition of apoptosis; ICAM — intercellular adhesion molecule; IL-1—interleukin 1; LPSlipopolysaccharide; MIP2—macrophage inflammatory protein 2; MMP-9 metalloproteinase-9; TNFR_tumor necrosis factor receptor. 